\title{
A Multilevel Thresholding Approach Based on Improved Particle Swarm Optimization for Color Image Segmentation
}

\author{
Larissa F. S. Britto ${ }^{1}$, Luciano D. S. Pacifico ${ }^{1}$, Teresa B. Ludermir ${ }^{2}$ \\ ${ }^{1}$ Departamento de Computação - Universidade Federal do Rural de Pernambuco \\ Recife - PE - Brazil \\ ${ }^{2}$ Centro de Informática - Universidade Federal de Pernambuco \\ Recife - PE - Brazil \\ \{larissa.feliciana, luciano.pacifico\}@ufrpe.br, tbl@cin.ufpe.br
}

\begin{abstract}
In this paper, a hybrid Otsu and improved Particle Swarm Optimization (PSO) algorithm is presented to deal with multilevel color image thresholding problem, named APSOW. In APSOW, the historical information represented by the local best solutions found so far by PSO population are permuted among the current population, using a randomized greedy process. APSOW also implements a weedout operator to prune the worst individuals from the population. The proposed APSOW is compared to other hybrid EAs and Otsu approaches from literature (include standard PSO model) through twelve benchmark color image problems, showing its potential and robustness.
\end{abstract}

\section{Introduction}

Image segmentation is one of the most fundamental processes in image understanding and computer vision. Image segmentation methods have two basic properties: discontinuity and similarity [Ye et al. 2015]. Thresholding is an important technique for image segmentation based on the similarity of image components. One of the most popular image thresholding methods is Otsu's algorithm [Otsu 1979].

The original Otsu algorithm was proposed as an effective method to segment an input image in gray level in two classes (image binarization). Otsu algorithm is easily adapted as multi-level thresholding approach, but multi-level Otsu has found little use in practice, since its computational cost increases significantly if the number of selected thresholds is high.

Recently, many Evolutionary Algorithms (EAs) and Swarm Intelligence (SIs) methods have been adapted to the context of multi-level image thresholding [Suresh and Lal 2017, Elaziz et al. 2019, Wang and Tan 2019]. In [Zhou and Yang 2011], Genetic Algorithm (GA) [Holland 1992] and Otsu have been used to infrared image segmentation. In [Kumar et al. 2011], Otsu algorithm have been combined to Differential Evolution (DE) [Storn and Price 1997] algorithm. In [Rodríguez-Esparza et al. 2020], Harris Hawks Optimization (HHO) haven been adapted to multi-level thresholding, using the Minimum Cross-Entropy as the fitness function. Particle Swarm Optimization (PSO) [Kennedy and Eberhart 1995] and Group Search Otpimization (GSO) [He et al. 2009] have also been hybridized with Otsu algorithm in [Zhang and Zhou 2012] and [Ye et al. 2015, Pacifico et al. 2018], respectively. 
In this work, we propose a hybrid improved PSO and Otsu algorithm, named APSOW, for color image segmentation problem based on multi-level image thresholding. The proposed APSOW performs a permutation on the historical information of the search (represented by the local best solutions found so far for each particle) in an attempt to speedup the convergence of the swarm, and also, to help slow particles to escape from local optima regions of the problem search space. APSOW also employs a weedout operator to prune a percentage of the weakest individuals from the population.

The paper is organized as follows. Otsu's algorithm, multi-level Otsu and PSO presented in Section 2. The proposed APSOW is presented in Section 3. The experimental results are discussed in Section 4. Finally, some conclusions and leads for future works are presented (Section 5).

\section{Background}

This section presents the background information concerning Otsu's algorithm and MultiLevel Otsu (Section 2.1), and also the main characteristics of standard PSO algorithm (Section 2.2). Brief descriptions for each model are presented as follows.

\subsection{Multi-Level Otsu Method}

The original Otsu method [Otsu 1979] aims to divide a given gray image $I$ in two classes $C_{0}$ and $C_{1}$ by the threshold $T$. But in multilevel Otsu, we consider that $D$ thresholds will be applied to partition $I$ in $(D+1)$ classes $C_{0}, C_{1}, \ldots, C_{D}$. Assuming that $I$ is represented in $L$ gray levels $[0,1, \ldots, L-1]$. Let $N_{p}$ denote the total number of pixels in $I$, and $n_{i}$ is the number of pixels at level $i$. The probability of gray level $i$ is denoted by eq. (1).

$$
p_{i}=n_{i} / N_{p}, p_{i} \geq 0, \sum_{i=0}^{L-1} p_{i}=1
$$

The gray level probability distribution for class $C_{k}$ is obtained by:

$$
r_{k}=\left\{\begin{array}{l}
\sum_{i=0}^{T_{i}} p_{i}, \text { if } k=0 \\
\sum_{i=T_{k}}^{T_{k}} p_{i}, \text { if } 1 \leq k \leq D-1 \\
\sum_{i=T_{D}}^{L-1} p_{i}, \text { if } k=D
\end{array}\right.
$$

where $T_{i}$ is $i$-th threshold, such that $T_{1}<T_{2}<\ldots<T_{D-1}<T_{D}$. The mean of class $C_{k}$ is denoted by:

$$
\mu_{k}=\left\{\begin{array}{l}
\left(\sum_{i=0}^{T_{i}} i \cdot p_{i}\right) / \sum_{i=0}^{T_{i}} p_{i}, \text { if } k=0 \\
\left(\sum_{i=T_{k}}^{T_{k}+1} i \cdot p_{i}\right) / \sum_{i=T_{k}}^{T_{k+1}} p_{i}, \text { if } 1 \leq k \leq D-1 \\
\left(\sum_{i=T_{D}}^{L-1} i \cdot p_{i}\right) / \sum_{i=T_{D}}^{L-1} p_{i}, \text { if } k=D
\end{array}\right.
$$

The Between-Class Variance (BCV) for multilevel Otsu is given by eq. (4) [Ye et al. 2015]. Multilevel Otsu algorithm finds the optimal set of thresholds by maximizing the BCV [Liu and Yu 2009].

$$
f_{I}=\sum_{i=0}^{D} r_{i} \cdot\left(\mu_{i}-\mu_{I}\right)^{2}, \mu_{I}=\sum_{i=0}^{D} r_{i} \mu_{i}
$$




\subsection{Particle Swarm Optimization}

Particle Swarm Optimization algorithm consists of a SI stochastic global search metaheuristic originated from the attempt to graphically simulate the social behavior of a flock of birds looking for resources [Kennedy and Eberhart 1995]. Later, looking for theoretical foundations, studies were realized concerning the way individuals in groups interact, exchanging information and reviewing personal concepts improving their adaptation to the environment [Kennedy et al. 2001]. In standard PSO, all individuals of the population follow the best point found so far by any individual of the population (global best or $g_{\text {best }}$ strategy).

In PSO, a population (swarm) $G$ of $S$ individuals (particles) is kept. Each particle keeps track of its position, velocity and best position. As the algorithm iterates, the velocity $\mathbf{V}_{i}^{t}$ of each particle $\mathbf{X}_{i}^{t}$ is determined according to the two main exemplar points of the search: the individual local best position visited so far $\mathbf{Y}_{i}^{t}$ and the global best position visited so far $\hat{\mathbf{Y}}^{t}$ by the swarm. The eq. (5) and eq. (6) describe respectively, how the new velocity and the new position of a particle are determined.

$$
\begin{gathered}
v_{i j}^{t+1}=w v_{i j}^{t}+c_{1} r_{1}\left(y_{i j}^{t}-x_{i j}^{t}\right)+c_{2} r_{2}\left(\hat{y}_{j}^{t}-x_{i j}^{t}\right) \\
x_{i j}^{t+1}=x_{i j}^{t}+v_{i j}^{t+1}
\end{gathered}
$$

where $1 \leq i \leq S, 1 \leq j \leq n, w$ is the scalar inertia weight (momentum term usually in the interval $[0.4,0.9])$, the values $r_{1}$ and $r_{2}$ are random variables taken from an uniform distribution $U(0,1)$, and the values $0<c_{1}, c_{2} \leq 2$ are individual and global acceleration coefficients, respectively. The new local best position $\mathbf{Y}_{i}^{t+1}$ and the new global best position $\hat{\mathbf{Y}}^{t+1}$ are determined by eq. (7) and eq. (8), respectively:

$$
\begin{gathered}
\mathbf{Y}_{i}^{t+1}=\left\{\begin{array}{l}
\mathbf{Y}_{i}^{t}, \text { if } f\left(\mathbf{Y}_{i}^{t+1}\right) \text { is better than } f\left(\mathbf{X}_{i}^{t}\right) \\
\mathbf{X}_{i}^{t+1}, \text { otherwise }
\end{array}\right. \\
\hat{\mathbf{Y}}^{t+1}=\arg \text { best }_{1 \leq i \leq S} f\left(\mathbf{Y}_{i}^{t+1}\right)
\end{gathered}
$$

\section{Proposed Approach: APSOW}

Let $I$ be a color image in RGB color space. The image $I$ is composed by three color channels, R, G and B. In APSOW, $I$ is decomposed for each color channel, generating the images $I_{R}, I_{G}$ and $I_{B}$ in gray level. Each gray level image is treated independently for segmentation purposes [Parthasarathy and Chitra 2015, Pacifico et al. 2018]. The $i$-th particle $\mathbf{X}_{i} \in \Re^{n}$ in APSOW represents $D$ dimensions (thresholds) for each RGB channel, where $n=3 \times D$. Fig. 1 represents an APSOW particle for $D=4$ (i.e., $\mathbf{X}_{i} \in \Re^{1} 2$ ), where the first four features represent the thresholds $T_{R 1}<T_{R 2}<T_{R 3}<T_{R 4}$ for $I_{R}$, the 5-th to 8-th features represent the thresholds $T_{G 1}<T_{G 2}<T_{G 3}<T_{G 4}$ for $I_{G}$ and the last four characteristics represent the thresholds $T_{B 1}<T_{B 2}<T_{B 3}<T_{B 4}$ for $I_{B}$.

The initial population for APSOW is randomly determined, respecting the restriction $T_{1}<T_{2}<\cdots<T_{D}$ for each RGB channel. After random initialization, the fitness 


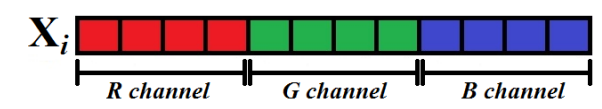

Figura 1. Particle representation for four thresholds per RGB channel.

function $f_{I}\left(\mathbf{X}_{i}^{0}\right)$ is computed for each particle $\mathbf{X}_{i}^{0}$ as:

$$
f_{I_{R G B}}\left(\mathbf{X}_{i}\right)=f_{I_{R}}\left(\mathbf{X}_{i}\right)+f_{I_{G}}\left(\mathbf{X}_{i}\right)+f_{I_{B}}\left(\mathbf{X}_{i}\right)
$$

After initialization, the generational process begins. At the beginning of each $t$-th generation in APSOW, the local best solutions for each image $\left(I_{R}, I_{G}\right.$ and $\left.I_{B}\right)$ are permuted among the particles using a greedy operator, in an attempt to speedup the convergence of the swarm and help weak particles to escape from local optma areas of the problem search space. Firstly, a random permutation is performed according to eq. (10):

$$
\mathbf{T} \mathbf{Y}^{t}=\text { permuting }\left(\mathbf{Y}^{t}\right)
$$

After that, each individual $\mathbf{T} \mathbf{Y}_{i}^{t}$ that represents a better solution than its corresponding particle $\mathbf{Y}_{i}^{t}$ will be used to replace $\mathbf{Y}_{i}^{t}$ in current local best population $\mathbf{Y}^{t}$ (eq. (11)).

$$
\mathbf{Y}_{i}^{t}=\left\{\begin{array}{l}
\mathbf{Y}_{i}^{t}, \text { if } f\left(\mathbf{Y}_{i}^{t+1}\right) \text { is better than } f\left(\mathbf{T} \mathbf{Y}_{i}^{t}\right) \\
\mathbf{T Y}_{i}^{t}, \text { otherwise }
\end{array}\right.
$$

Once the $\mathbf{Y}^{t}$ is defined, PSO operators are executed as in standard PSO. In each generation, after the evaluation of the new swarm $G^{t+1}$, a selection operator to prune the weakest particles from the current swarm. A percentage of the weakest particles for each image $I_{R}, I_{G}$ and $I_{B}$ will be selected to be pruned by random reinitialization according to the partial fitness function $f_{R}\left(\mathbf{X}_{i}^{t}\right), f_{G}\left(\mathbf{X}_{i}^{t}\right)$ and $f_{B}\left(\mathbf{X}_{i}^{t}\right)$. Since each RGB channel is evaluated independently, one particle $\mathbf{X}_{i}^{t}$ may be considered weak for some RGB channel but not weak considering the other channels. In this situation, only the characteristics from $\mathbf{X}_{i}^{t}$ corresponding to the weak channels are pruned. This operator aims to promote a better escaping from local minima points. The algorithm for APSOW is presented in Algorithm 1.

\section{Experimental Results}

In this section, the proposed APSOW is tested by means of twelve benchmark color images using four other EAs from literature as comparison methods: GA, DE, PSO and GSO. The selected benchmark color images are presented inf Fig. 2. The parameters for each algorithm are presented in Table 1, according to [Ye et al. 2015, Pacifico et al. 2018].

All algorithms have been run in MATLAB 7.6 environment, and all tests have been executed in a computer with an i5-5250U CPU and 8 GB RAM. In the experiments, the dimension of thresholding value is set from 2 to 5 for each RGB channel [Ye et al. 2015]. For each test, thirty independent executions have been run for each color image. All evolutionary algorithms have started with the same initial population in each test. The initial population has been acquired by random sampling, where, for each RGB channel, all thresholds have been randomly determined and sorted, so we have $T_{1}<T_{2}<\cdots<T_{D}$. 


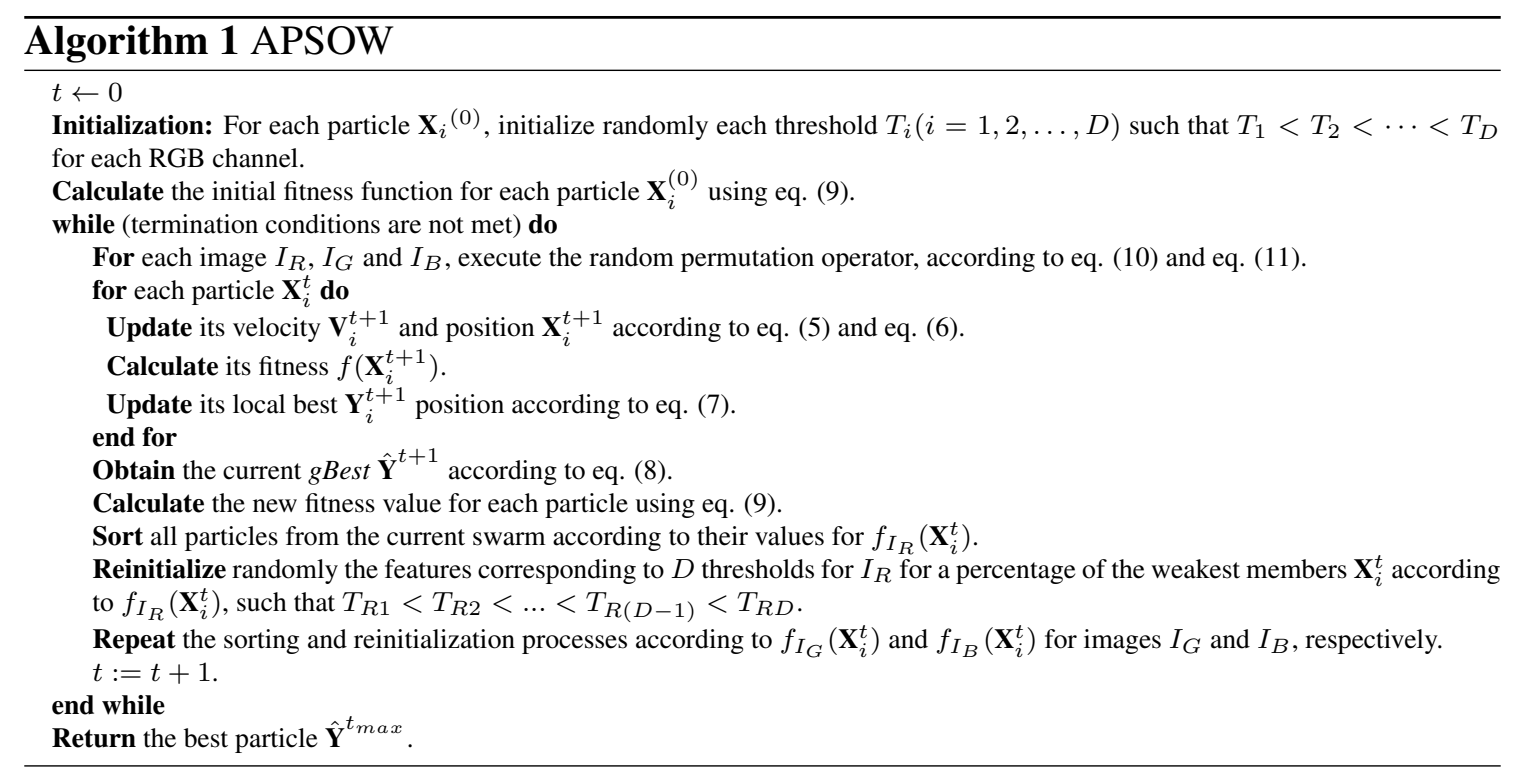

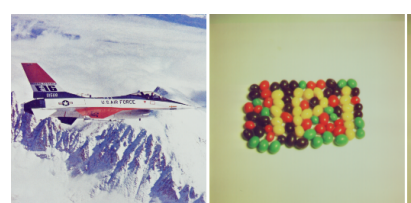

(a)

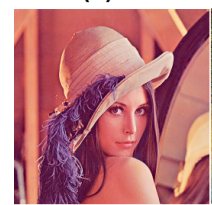

(g) (b)

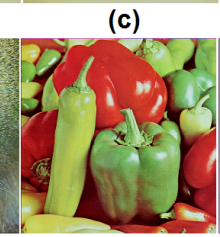

(h)

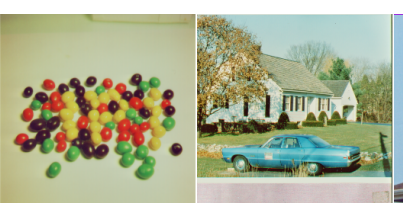

(d)

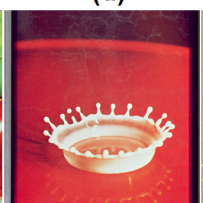

(j)

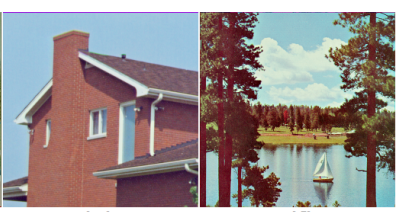

(e) (f)

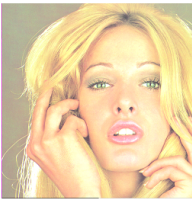

(k)

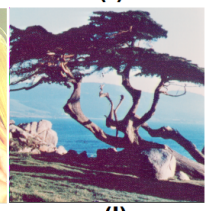

(I)

Figura 2. Benchmark color images: (a) Airplane, (b) Gellybeans1, (c) Geallybeans2, (d) House1, (e) House2, (f) Lake, (g) Lena, (h) Mandrill, (i) Peppers, (j) Splash, (k) Tiffany and (I) Tree.

Tabela 1. Parameters for each algorithm.

\begin{tabular}{|c|c|c|}
\hline Algorithm & Parameter & Value \\
\hline \multirow{2}{*}{ All EAs } & $S$ & 20 \\
& $t_{\max }$ & 100 \\
\hline GA & $C_{r}$ and $M_{r}$ & 0.8 and 0.1 \\
\hline DE & $C_{r}$ and $F$ & 0.3 and 0.5 \\
\hline PSO-based Approaches & $c_{1}$ and $c_{2}$ & 2.0 \\
& $w$ & 0.9 to 0.4 \\
\hline GSO & $l_{m}$ ax and $\theta_{\max }$ & 5 and 5 \\
\hline APSOW & Weedout Rate & $20 \%$ \\
\hline
\end{tabular}




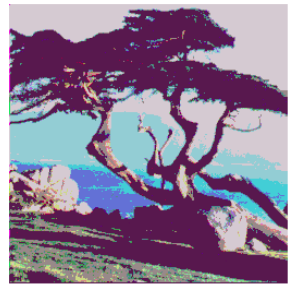

(a)

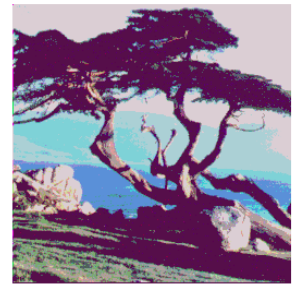

(b)

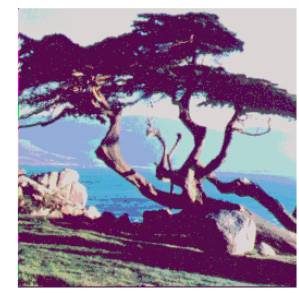

(c)

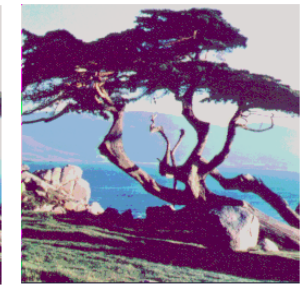

(d)

Figura 3. APSOW sample results for Tree image: (a) 2-D, (b) 3-D, (c) 4-D, (d) 5-D.

The evaluation criterion includes an overall rank system employed through the application of Friedman hypothesis test [Friedman 1937], adopting Nemenyi test as the post-hoc test, in relation to the fitness function (eq. (9)) [Demšar 2006] and its components to each RGB channel (images $I_{R}, I_{G}$ and $I_{B}$ ). The experimental results are shown from Table 2 to Table 5. Some sample results for the proposed APSOW are shown in Fig. 3.

The overall evaluation performed through the application of the Friedman tests are presented in Table 6. The overall evaluation shows that the proposed APSOW is able to find the best performances according to the ranking system for $D=2,3$ and 5 (except for $I_{G}$ when $D=3$, where it has achieved the second best value), with statistical significance in relation to GA and GSO, and it has outperformed PSO and DE with statistical significance in most of the cases. For $D=4$, APSOW has been able to find the best ranks for $I_{B}$ and considering the whole image $I$, reaching the second best performances in relation to $I_{R}$ and $I_{B}$. The overall evaluation showed the potential and robustness of the proposed APSOW to tackle image segmentation problems.

The evaluation considering the average execution times (see Table 2 to Table 5) shows that the execution time of APSOW is slightly higher than standard PSO, as expected, but its average execution times are completely compatible with the execution times of any evolutionary and swarm intelligence algorithms, making APSOW an interesting option to deal with image thresholding applications.

\section{Conclusions}

In this work, we presented a new hybrid improved PSO and Otsu algorithm for multi-level color image thresholding, called APSOW. APSOW employs a permutation to randomize the historical information stored in local best population to speedup the swarm convergence, an a weedout operator to prune a percentage of the weakest particles from the swarm, promoting a better escaping mechanism from local minima points.

Experimental evaluation has been conducted using twelve benchmark color images and by the comparison of proposed APSOW in relation to four EAs from the literature: GA, DE, standard PSO and GSO. The experimental results have been evaluated by hypothesis tests of type Friedman test in relation to the Between-Class Variance index (the adopted fitness function for all evaluated EAs), considering the original color image (in RGB) and each one its color channels individually. According to Friedman/Nemenyi tests, the proposed APSOW is able to outperform the comparison approaches in most of the evaluated scenarios, showing the potential of the proposed method for image segmentation tasks. 
Tabela 2. Experimental results for 2-D: Mean \pm standard deviation.

\begin{tabular}{|c|c|c|c|c|c|c|}
\hline Image & Algorithm & $f_{I_{R}}$ & $f_{I_{G}}$ & $f_{I_{B}}$ & $f_{I}$ & Time (s) \\
\hline \multirow{5}{*}{ Airplane } & GA & $1763.1 \pm 19.83$ & $2403.3 \pm 20.11$ & $906.35 \pm 23.95$ & $5072.7 \pm 30.00$ & $2.3797 \pm 0.0160$ \\
\hline & $\mathrm{DE}$ & $1787.9 \pm 0.0053$ & $2436.4 \pm 0.0006$ & $930.78 \pm 0.0032$ & $5155.1 \pm 0.0061$ & $2.3459 \pm 0.0157$ \\
\hline & PSO & $1787.9 \pm 0.0087$ & $2436.4 \pm 0.0004$ & $930.78 \pm 0.0$ & $5155.1 \pm 0.0087$ & $2.3319 \pm 0.0249$ \\
\hline & GSO & $1787.9 \pm 0.2361$ & $2436.3 \pm 0.0959$ & $925.51 \pm 27.23$ & $5149.7 \pm 27.24$ & $2.4438 \pm 0.0216$ \\
\hline & APSOW & $1787.9 \pm 0.0052$ & $2436.4 \pm 0.0002$ & $9307.8 \pm 0.0$ & $5155.1 \pm 0.0052$ & $2.4796 \pm 0.0668$ \\
\hline \multirow{5}{*}{ Gellybeans 1} & GA & $1034.8 \pm 15.50$ & $1883.8 \pm 16.00$ & $1641.0 \pm 11.13$ & $4559.6 \pm 13.02$ & $0.8600 \pm 0.0242$ \\
\hline & $\mathrm{DE}$ & $1052.5 \pm 0.0027$ & $1904.2 \pm 0.0075$ & $1653.9 \pm 0.0056$ & $4610.5 \pm 0.0117$ & $0.8256 \pm 0.0242$ \\
\hline & PSO & $1050.8 \pm 9.197$ & $1904.2 \pm 0.0008$ & $1653.9 \pm 0.0064$ & $4608.8 \pm 9.197$ & $0.8172 \pm 0.0242$ \\
\hline & GSO & $1047.4 \pm 15.37$ & $1904.2 \pm 0.0$ & $1648.1 \pm 31.54$ & $4599.7 \pm 34.22$ & $0.9354 \pm 0.0262$ \\
\hline & APSOW & $1052.5 \pm 0.0065$ & $1904.2 \pm 0.0011$ & $1653.9 \pm 0.0052$ & $4610.5 \pm 0.0105$ & $0.8452 \pm 0.0085$ \\
\hline \multirow{5}{*}{ Gellybeans2 } & GA & $1374.2 \pm 22.79$ & $2374.5 \pm 22.33$ & $1940.5 \pm 20.47$ & $5689.2 \pm 28.30$ & $0.8573 \pm 0.0171$ \\
\hline & $\mathrm{DE}$ & $1398.3 \pm 0.0035$ & $2401.1 \pm 0.0$ & $1969.7 \pm 0.0$ & $5769.1 \pm 0.0035$ & $0.8261 \pm 0.0241$ \\
\hline & PSO & $1395.4 \pm 16.20$ & $2401.1 \pm 0.0103$ & $1969.7 \pm 0.0$ & $5766.1 \pm 16.20$ & $0.8182 \pm 0.0232$ \\
\hline & GSO & $1394.1 \pm 16.52$ & $2401.1 \pm 0.1291$ & $1969.6 \pm 0.2913$ & $5764.8 \pm 16.89$ & $0.9395 \pm 0.0291$ \\
\hline & APSOW & $1398.3 \pm 0.0035$ & $2401.1 \pm 0.0103$ & $1969.7 \pm 0.0069$ & $5769.1 \pm 0.0123$ & $0.8461 \pm 0.0069$ \\
\hline \multirow{5}{*}{ House 1} & GA & $2173.4 \pm 45.18$ & $1971.5 \pm 39.97$ & $3369.8 \pm 36.38$ & $7514.7 \pm 64.99$ & $2.3902 \pm 0.0662$ \\
\hline & $\mathrm{DE}$ & $2223.4 \pm 0.0$ & $2023.9 \pm 0.0090$ & $3413.0 \pm 0.0$ & $7660.2 \pm 0.0090$ & $2.3464 \pm 0.0137$ \\
\hline & PSO & $2223.4 \pm 0.0097$ & $2023.9 \pm 0.0090$ & $3413.0 \pm 0.0162$ & $7660.2 \pm 0.0197$ & $2.3360 \pm 0.0135$ \\
\hline & GSO & $2223.4 \pm 0.0239$ & $2023.9 \pm 0.0$ & $3413.0 \pm 0.0182$ & $7660.2 \pm 0.0311$ & $2.4438 \pm 0.0166$ \\
\hline & APSOW & $2223.4 \pm 0.0297$ & $2023.9 \pm 0.0$ & $3413.0 \pm 0.0182$ & $7660.2 \pm 0.0324$ & $2.4641 \pm 0.0164$ \\
\hline \multirow{5}{*}{ House 2} & GA & $816.19 \pm 19.10$ & $2945.8 \pm 20.61$ & $3754.6 \pm 15.21$ & $7516.5 \pm 24.68$ & $0.8656 \pm 0.0340$ \\
\hline & $\mathrm{DE}$ & $843.20 \pm 4.442$ & $2965.4 \pm 0.0902$ & $3769.7 \pm 0.5307$ & $7578.3 \pm 4.479$ & $0.8177 \pm 0.0189$ \\
\hline & PSO & $834.47 \pm 8.845$ & $2965.4 \pm 0.0045$ & $3769.7 \pm 0.5375$ & $7569.5 \pm 8.968$ & $0.8255 \pm 0.0416$ \\
\hline & GSO & $836.22 \pm 8.905$ & $2965.4 \pm 0.0$ & $3769.7 \pm 0.5385$ & $7571.3 \pm 8.955$ & $0.9491 \pm 0.0576$ \\
\hline & APSOW & $844.41 \pm 0.0114$ & $2965.4 \pm 0.0089$ & $3769.5 \pm 0.5108$ & $7579.4 \pm 0.5103$ & $0.8579 \pm 0.0137$ \\
\hline \multirow{5}{*}{ Lake } & GA & $1548.6 \pm 30.11$ & $5595.0 \pm 34.09$ & $5599.2 \pm 36.65$ & $12742.7 \pm 48.61$ & $2.3840 \pm 0.0424$ \\
\hline & $\mathrm{DE}$ & $1589.9 \pm 0.0042$ & $5636.6 \pm 0.0$ & $5640.5 \pm 0.0104$ & $12867.0 \pm 0.0132$ & $2.3542 \pm 0.0729$ \\
\hline & PSO & $1589.9 \pm 0.0$ & $5636.6 \pm 0.0083$ & $5640.5 \pm 0.0062$ & $12867.0 \pm 0.0102$ & $2.3355 \pm 0.0177$ \\
\hline & GSO & $1589.9 \pm 0.0042$ & $5636.6 \pm 0.0$ & $5640.5 \pm 0.0$ & $12867.0 \pm 0.0042$ & $2.4881 \pm 0.0952$ \\
\hline & APSOW & $1589.9 \pm 0.0042$ & $5636.6 \pm 0.0115$ & $5640.5 \pm 0.0062$ & $12867.0 \pm 0.0132$ & $2.4858 \pm 0.0238$ \\
\hline \multirow{5}{*}{ Lena } & GA & $2140.5 \pm 35.25$ & $2328.9 \pm 44.02$ & $956.20 \pm 53.37$ & $5425.6 \pm 67.83$ & $2.3729 \pm 0.0122$ \\
\hline & $\mathrm{DE}$ & $2183.7 \pm 0.0$ & $2393.6 \pm 0.0$ & $1004.9 \pm 0.0$ & $5582.3 \pm 0.0$ & $2.3683 \pm 0.0322$ \\
\hline & PSO & $2183.7 \pm 0.0163$ & $2393.6 \pm 0.0079$ & $1004.9 \pm 0.0$ & $5582.3 \pm 0.0178$ & $2.3349 \pm 0.0497$ \\
\hline & GSO & $2183.7 \pm 0.0276$ & $2393.6 \pm 0.0$ & $1004.9 \pm 0.0$ & $5582.3 \pm 0.0276$ & $2.4688 \pm 0.0591$ \\
\hline & APSOW & $2183.7 \pm 0.0117$ & $2393.6 \pm 0.0$ & $1004.9 \pm 0.0150$ & $5582.3 \pm 0.0187$ & $2.4646 \pm 0.0147$ \\
\hline \multirow{5}{*}{ Mandril } & GA & $2584.3 \pm 39.10$ & $1946.4 \pm 40.56$ & $3295.5 \pm 60.86$ & $7826.1 \pm 66.89$ & $2.3880 \pm 0.0275$ \\
\hline & DE & $2642.2 \pm 0.0112$ & $2004.7 \pm 0.0138$ & $3360.0 \pm 0.0064$ & $8006.9 \pm 0.0169$ & $2.3463 \pm 0.0130$ \\
\hline & PSO & $2642.2 \pm 0.0081$ & $2004.7 \pm 0.0138$ & $3360.0 \pm 0.0150$ & $8006.9 \pm 0.0242$ & $2.3496 \pm 0.0242$ \\
\hline & GSO & $2642.2 \pm 0.1318$ & $2004.7 \pm 0.0641$ & $3360.0 \pm 0.0246$ & $8006.9 \pm 0.2169$ & $2.5016 \pm 0.0891$ \\
\hline & APSOW & $2642.2 \pm 0.0081$ & $2004.7 \pm 0.0147$ & $3360.0 \pm 0.0150$ & $8006.9 \pm 0.0189$ & $2.4791 \pm 0.0143$ \\
\hline \multirow{5}{*}{ Peppers } & GA & $1710.7 \pm 33.16$ & $5146.3 \pm 43.45$ & $1611.6 \pm 35.25$ & $8468.5 \pm 40.41$ & $2.4016 \pm 0.0799$ \\
\hline & $\mathrm{DE}$ & $1759.2 \pm 0.0$ & $5203.9 \pm 0.0$ & $1656.2 \pm 0.0$ & $8619.3 \pm 0.0$ & $2.3407 \pm 0.0118$ \\
\hline & PSO & $1759.2 \pm 0.0173$ & $5203.9 \pm 0.0110$ & $1656.2 \pm 0.0117$ & $8619.3 \pm 0.0229$ & $2.3422 \pm 0.0284$ \\
\hline & GSO & $1759.2 \pm 0.0185$ & $5203.9 \pm 0.0228$ & $1656.2 \pm 0.0$ & $8619.3 \pm 0.0281$ & $2.4646 \pm 0.0287$ \\
\hline & APSOW & $1759.2 \pm 0.0149$ & $5203.9 \pm 0.0184$ & $1656.2 \pm 0.0$ & $8619.3 \pm 0.0229$ & $2.4802 \pm 0.0429$ \\
\hline \multirow{5}{*}{ Splash } & GA & $3244.5 \pm 19.45$ & $3170.7 \pm 23.00$ & $2005.9 \pm 30.94$ & $8421.1 \pm 33.84$ & $2.3777 \pm 0.0229$ \\
\hline & $\mathrm{DE}$ & $3268.2 \pm 0.0502$ & $3206.0 \pm 6.797$ & $2039.4 \pm 0.0263$ & $8513.5 \pm 6.789$ & $2.3338 \pm 0.0096$ \\
\hline & PSO & $3268.2 \pm 0.0$ & $3183.9 \pm 17.07$ & $2039.4 \pm 0.0$ & $8491.5 \pm 17.07$ & $2.3220 \pm 0.0171$ \\
\hline & GSO & $3263.5 \pm 25.72$ & $3185.1 \pm 17.45$ & $2039.4 \pm 0.0$ & $8488.0 \pm 33.06$ & $2.4802 \pm 0.0466$ \\
\hline & APSOW & $3268.2 \pm 0.0029$ & $3207.6 \pm 0.0$ & $2039.4 \pm 0.0115$ & $8515.2 \pm 0.0117$ & $2.4875 \pm 0.0656$ \\
\hline \multirow{5}{*}{ Tiffany } & GA & $846.46 \pm 8.954$ & $865.38 \pm 9.889$ & $464.44 \pm 12.85$ & $2176.3 \pm 13.31$ & $2.3918 \pm 0.0536$ \\
\hline & $\mathrm{DE}$ & $861.34 \pm 2.512$ & $883.93 \pm 3.505$ & $481.46 \pm 0.5415$ & $2226.7 \pm 3.787$ & $2.3500 \pm 0.0568$ \\
\hline & PSO & $854.43 \pm 6.917$ & $880.53 \pm 6.572$ & $469.47 \pm 22.68$ & $2204.4 \pm 22.92$ & $2.3167 \pm 0.0092$ \\
\hline & GSO & $851.01 \pm 8.626$ & $867.10 \pm 16.32$ & $464.19 \pm 25.27$ & $2182.3 \pm 32.88$ & $2.4614 \pm 0.0419$ \\
\hline & APSOW & $861.12 \pm 3.321$ & $885.80 \pm 0.0422$ & $481.76 \pm 0.0107$ & $2228.7 \pm 3.315$ & $2.5302 \pm 0.1415$ \\
\hline \multirow{5}{*}{ Tree } & GA & $2983.5 \pm 36.72$ & $5494.9 \pm 32.69$ & $3763.3 \pm 38.78$ & $12241.7 \pm 58.70$ & $0.8807 \pm 0.0276$ \\
\hline & $\mathrm{DE}$ & $3031.4 \pm 0.0$ & $5530.6 \pm 0.0$ & $3806.7 \pm 0.0$ & $12368.7 \pm 0.0$ & $0.8189 \pm 0.0182$ \\
\hline & PSO & $3031.4 \pm 0.0$ & $5530.6 \pm 0.0$ & $3806.7 \pm 0.0122$ & $12368.7 \pm 0.0122$ & $0.8067 \pm 0.0088$ \\
\hline & GSO & $3031.4 \pm 0.0$ & $5530.6 \pm 0.0$ & $3806.7 \pm 0.0167$ & $12368.7 \pm 0.0167$ & $0.9344 \pm 0.0150$ \\
\hline & APSOW & $3031.4 \pm 0.0$ & $5530.6 \pm 0.0$ & $3806.7 \pm 0.0147$ & $12368.7 \pm 0.0147$ & $0.8583 \pm 0.0099$ \\
\hline
\end{tabular}


Tabela 3. Experimental results for 3-D: Mean \pm standard deviation.

\begin{tabular}{|c|c|c|c|c|c|c|}
\hline Image & Algorithm & $f_{I_{R}}$ & $f_{I_{G}}$ & $f_{I_{B}}$ & $f_{I}$ & Time (s) \\
\hline \multirow{5}{*}{ Airplane } & GA & $1827.9 \pm 16.39$ & $2508.7 \pm 18.40$ & $948.54 \pm 17.31$ & $5285.1 \pm 19.02$ & $2.4828 \pm 0.0227$ \\
\hline & $\mathrm{DE}$ & $1855.6 \pm 0.6661$ & $2533.4 \pm 0.7497$ & $970.29 \pm 0.7321$ & $5359.3 \pm 1.261$ & $2.4433 \pm 0.0138$ \\
\hline & PSO & $1856.3 \pm 0.3349$ & $2534.0 \pm 0.1129$ & $969.51 \pm 7.401$ & $5359.8 \pm 7.379$ & $2.4375 \pm 0.0270$ \\
\hline & GSO & $1853.4 \pm 12.40$ & $2533.8 \pm 0.6388$ & $960.97 \pm 14.83$ & $5348.2 \pm 18.32$ & $2.5954 \pm 0.0217$ \\
\hline & APSOW & $1856.3 \pm 0.0861$ & $2534.0 \pm 0.0986$ & $971.07 \pm 0.0789$ & $5361.4 \pm 0.1805$ & $2.5731 \pm 0.0189$ \\
\hline \multirow{5}{*}{ Gellybeans 1} & GA & $1054.4 \pm 10.45$ & $1937.9 \pm 9.369$ & $1672.8 \pm 10.55$ & $4665.0 \pm 13.65$ & $0.9459 \pm 0.0469$ \\
\hline & $\mathrm{DE}$ & $1075.4 \pm 0.6677$ & $1952.9 \pm 0.4598$ & $1689.9 \pm 0.3213$ & $4718.2 \pm 0.9855$ & $0.8948 \pm 0.0313$ \\
\hline & PSO & $1074.3 \pm 5.947$ & $1953.1 \pm 0.0593$ & $1684.8 \pm 10.81$ & $4712.2 \pm 12.01$ & $0.8938 \pm 0.0270$ \\
\hline & GSO & $1074.5 \pm 4.250$ & $1952.5 \pm 1.235$ & $1682.5 \pm 11.80$ & $4709.5 \pm 11.89$ & $1.0865 \pm 0.0320$ \\
\hline & APSOW & $1075.9 \pm 0.0611$ & $1953.1 \pm 0.0569$ & $1689.2 \pm 2.191$ & $4718.2 \pm 2.193$ & $0.9141 \pm 0.0127$ \\
\hline \multirow{5}{*}{ Gellybeans2 } & GA & $1405.4 \pm 19.33$ & $2440.0 \pm 15.30$ & $1991.4 \pm 16.68$ & $5836.8 \pm 14.61$ & $0.9349 \pm 0.0243$ \\
\hline & $\mathrm{DE}$ & $1431.5 \pm 0.3986$ & $2465.2 \pm 0.4082$ & $2015.7 \pm 1.318$ & $5912.4 \pm 1.467$ & $0.8912 \pm 0.0211$ \\
\hline & PSO & $1428.5 \pm 10.22$ & $2465.5 \pm 0.0842$ & $2010.0 \pm 16.14$ & $5904.0 \pm 17.84$ & $0.8897 \pm 0.0232$ \\
\hline & GSO & $1430.5 \pm 6.090$ & $2465.3 \pm 0.4169$ & $2011.1 \pm 11.53$ & $5907.0 \pm 12.92$ & $1.0802 \pm 0.0309$ \\
\hline & APSOW & $1431.8 \pm 0.0520$ & $2465.5 \pm 0.0609$ & $2016.5 \pm 0.0639$ & $5913.8 \pm 0.1147$ & $0.9240 \pm 0.0223$ \\
\hline \multirow{5}{*}{ House1 } & GA & $2308.9 \pm 45.35$ & $2123.5 \pm 42.61$ & $3520.3 \pm 43.94$ & $7952.6 \pm 45.50$ & $2.4917 \pm 0.0467$ \\
\hline & $\mathrm{DE}$ & $2369.6 \pm 0.3048$ & $2171.9 \pm 0.4432$ & $3575.6 \pm 0.1726$ & $8117.1 \pm 0.5222$ & $2.4298 \pm 0.0140$ \\
\hline & PSO & $2369.8 \pm 0.0964$ & $2172.1 \pm 0.0457$ & $3575.7 \pm 0.0465$ & $8117.7 \pm 0.1218$ & $2.4355 \pm 0.0494$ \\
\hline & GSO & $2369.8 \pm 0.2255$ & $2172.0 \pm 0.2266$ & $3570.3 \pm 29.72$ & $8112.1 \pm 29.69$ & $2.6109 \pm 0.0804$ \\
\hline & APSOW & $2369.8 \pm 0.0923$ & $2172.0 \pm 0.1270$ & $3575.7 \pm 0.0554$ & $8117.6 \pm 0.1888$ & $2.5600 \pm 0.0259$ \\
\hline \multirow{5}{*}{ House 2} & GA & $886.40 \pm 24.08$ & $2991.7 \pm 19.09$ & $3822.4 \pm 20.61$ & $7700.5 \pm 23.05$ & $0.9391 \pm 0.0194$ \\
\hline & $\mathrm{DE}$ & $918.86 \pm 0.0405$ & $3023.5 \pm 0.0277$ & $3855.1 \pm 0.0544$ & $7797.5 \pm 0.0901$ & $0.8939 \pm 0.0219$ \\
\hline & PSO & $918.86 \pm 0.0151$ & $3023.5 \pm 0.0191$ & $3849.4 \pm 21.54$ & $7791.8 \pm 21.53$ & $0.8943 \pm 0.0294$ \\
\hline & GSO & $909.37 \pm 24.11$ & $3015.2 \pm 11.82$ & $3852.2 \pm 15.53$ & $7776.8 \pm 31.75$ & $1.0766 \pm 0.0226$ \\
\hline & APSOW & $918.86 \pm 0.0207$ & $3023.5 \pm 0.0508$ & $3855.1 \pm 0.0379$ & $7797.4 \pm 0.0702$ & $0.9198 \pm 0.0114$ \\
\hline \multirow{5}{*}{ Lake } & GA & $1651.2 \pm 25.36$ & $5736.9 \pm 23.73$ & $5703.3 \pm 30.49$ & $13091.4 \pm 32.10$ & $2.4880 \pm 0.0628$ \\
\hline & $\mathrm{DE}$ & $1693.7 \pm 0.4003$ & $5776.3 \pm 0.4174$ & $5739.9 \pm 0.2470$ & $13209.9 \pm 0.7330$ & $2.4391 \pm 0.0186$ \\
\hline & PSO & $1687.1 \pm 26.42$ & $5776.7 \pm 0.0543$ & $5740.3 \pm 0.0755$ & $13204.0 \pm 26.41$ & $2.4276 \pm 0.0088$ \\
\hline & GSO & $1690.5 \pm 19.01$ & $5775.8 \pm 1.909$ & $5739.4 \pm 1.576$ & $13205.8 \pm 18.95$ & $2.6068 \pm 0.0314$ \\
\hline & APSOW & $1694.0 \pm 0.1277$ & $5776.6 \pm 0.1894$ & $5740.2 \pm 0.1274$ & $13210.7 \pm 0.3213$ & $2.5684 \pm 0.0357$ \\
\hline \multirow{5}{*}{ Lena } & GA & $2227.4 \pm 28.53$ & $2549.6 \pm 24.12$ & $1014.4 \pm 28.45$ & $5791.4 \pm 34.59$ & $2.4818 \pm 0.0207$ \\
\hline & $\mathrm{DE}$ & $2272.3 \pm 0.2780$ & $2597.8 \pm 0.1679$ & $1069.9 \pm 0.2474$ & $5940.0 \pm 0.4014$ & $2.4417 \pm 0.0711$ \\
\hline & PSO & $2263.5 \pm 27.08$ & $2591.1 \pm 37.30$ & $1061.4 \pm 22.53$ & $5916.1 \pm 47.13$ & $2.4318 \pm 0.0662$ \\
\hline & GSO & $2262.9 \pm 26.86$ & $2597.9 \pm 0.1255$ & $1056.0 \pm 24.83$ & $5916.8 \pm 32.96$ & $2.6109 \pm 0.0182$ \\
\hline & APSOW & $2272.4 \pm 0.2046$ & $2597.9 \pm 0.0755$ & $1070.0 \pm 0.1244$ & $5940.4 \pm 0.2826$ & $2.5536 \pm 0.0246$ \\
\hline \multirow{5}{*}{ Mandril } & GA & $2766.8 \pm 41.40$ & $2055.0 \pm 40.72$ & $3501.8 \pm 43.47$ & $8323.6 \pm 33.76$ & $2.5089 \pm 0.0284$ \\
\hline & $\mathrm{DE}$ & $2830.3 \pm 0.3190$ & $2113.2 \pm 0.4475$ & $3555.6 \pm 0.2109$ & $8499.2 \pm 0.6765$ & $2.4532 \pm 0.0188$ \\
\hline & PSO & $2830.6 \pm 0.0482$ & $2110.0 \pm 19.89$ & $3555.8 \pm 0.1699$ & $8496.4 \pm 19.87$ & $2.4370 \pm 0.0111$ \\
\hline & GSO & $2830.6 \pm 0.1471$ & $2113.4 \pm 0.7137$ & $3555.8 \pm 0.1919$ & $8499.8 \pm 0.8534$ & $2.6402 \pm 0.0530$ \\
\hline & APSOW & $2830.5 \pm 0.2417$ & $2113.5 \pm 0.2280$ & $3555.7 \pm 0.1694$ & $8499.7 \pm 0.4823$ & $2.5771 \pm 0.0311$ \\
\hline \multirow{5}{*}{ Peppers } & GA & $1822.5 \pm 42.87$ & $5324.6 \pm 34.07$ & $1725.3 \pm 31.76$ & $8872.3 \pm 32.62$ & $2.4787 \pm 0.0265$ \\
\hline & $\mathrm{DE}$ & $1884.5 \pm 1.134$ & $5381.1 \pm 3.245$ & $1770.7 \pm 11.49$ & $9036.4 \pm 11.28$ & $2.4604 \pm 0.0545$ \\
\hline & PSO & $1881.2 \pm 23.04$ & $5382.5 \pm 0.0664$ & $1773.0 \pm 10.55$ & $9036.8 \pm 24.60$ & $2.4235 \pm 0.0222$ \\
\hline & GSO & $1885.4 \pm 0.2749$ & $5378.8 \pm 11.07$ & $1754.5 \pm 23.91$ & $9018.7 \pm 25.08$ & $2.6114 \pm 0.0219$ \\
\hline & APSOW & $1885.4 \pm 0.0985$ & $5382.5 \pm 0.1394$ & $1777.1 \pm 0.0833$ & $9045.0 \pm 0.2260$ & $2.5714 \pm 0.0323$ \\
\hline \multirow{5}{*}{ Splash } & GA & $3366.1 \pm 39.74$ & $3423.3 \pm 27.74$ & $2044.0 \pm 26.87$ & $8833.3 \pm 35.94$ & $2.4928 \pm 0.0382$ \\
\hline & $\mathrm{DE}$ & $3413.9 \pm 0.1396$ & $3453.0 \pm 0.1008$ & $2081.0 \pm 1.193$ & $8948.0 \pm 1.079$ & $2.4474 \pm 0.0352$ \\
\hline & PSO & $3414.0 \pm 0.0131$ & $3453.1 \pm 0.0189$ & $2077.8 \pm 10.50$ & $8944.9 \pm 10.50$ & $2.4198 \pm 0.0100$ \\
\hline & GSO & $3412.0 \pm 11.14$ & $3447.5 \pm 30.68$ & $2080.4 \pm 2.394$ & $8939.9 \pm 32.48$ & $2.6229 \pm 0.0478$ \\
\hline & APSOW & $3414.0 \pm 0.0849$ & $3453.1 \pm 0.0420$ & $2080.8 \pm 1.353$ & $8947.9 \pm 1.324$ & $2.5709 \pm 0.0498$ \\
\hline \multirow{5}{*}{ Tiffany } & GA & $883.08 \pm 12.42$ & $971.56 \pm 11.59$ & $496.72 \pm 11.70$ & $2351.4 \pm 13.45$ & $2.4938 \pm 0.0282$ \\
\hline & $\mathrm{DE}$ & $905.57 \pm 2.936$ & $987.90 \pm 0.3377$ & $521.74 \pm 0.5117$ & $2415.2 \pm 2.944$ & $2.4230 \pm 0.0101$ \\
\hline & PSO & $892.51 \pm 12.96$ & $984.96 \pm 7.164$ & $511.11 \pm 9.761$ & $2388.6 \pm 16.49$ & $2.4173 \pm 0.0158$ \\
\hline & GSO & $8778.0 \pm 18.88$ & $974.35 \pm 28.03$ & $507.28 \pm 17.47$ & $2359.4 \pm 35.13$ & $2.6031 \pm 0.0106$ \\
\hline & APSOW & $905.71 \pm 2.956$ & $987.98 \pm 0.2496$ & $522.05 \pm 0.0977$ & $2415.7 \pm 2.952$ & $2.5605 \pm 0.0217$ \\
\hline \multirow{5}{*}{ Tree } & GA & $3075.8 \pm 27.92$ & $5632.8 \pm 25.59$ & $3838.0 \pm 23.37$ & $12546.7 \pm 27.71$ & $0.9698 \pm 0.0303$ \\
\hline & $\mathrm{DE}$ & $3109.9 \pm 0.7436$ & $5662.7 \pm 0.5664$ & $3871.9 \pm 0.4114$ & $12644.5 \pm 1.212$ & $0.8876 \pm 0.0105$ \\
\hline & PSO & $3108.8 \pm 9.552$ & $5663.2 \pm 0.0976$ & $3865.3 \pm 15.87$ & $12637.3 \pm 21.62$ & $0.8802 \pm 0.0104$ \\
\hline & GSO & $3103.7 \pm 15.28$ & $5663.2 \pm 0.1161$ & $3866.6 \pm 14.41$ & $12633.5 \pm 22.94$ & $1.0703 \pm 0.0090$ \\
\hline & APSOW & $3110.6 \pm 0.0610$ & $5663.1 \pm 0.1387$ & $3872.3 \pm 0.1467$ & $12646.0 \pm 0.2657$ & $0.9318 \pm 0.0149$ \\
\hline
\end{tabular}


Tabela 4. Experimental results for 4-D: Mean \pm standard deviation.

\begin{tabular}{|c|c|c|c|c|c|c|}
\hline Image & Algorithm & $f_{I_{R}}$ & $f_{I_{G}}$ & $f_{I_{B}}$ & $f_{I}$ & Time (s) \\
\hline \multirow{5}{*}{ Airplane } & GA & $1871.5 \pm 18.66$ & $255.45 \pm 17.10$ & $963.03 \pm 16.65$ & $5389.1 \pm 16.73$ & $2.5365 \pm 0.0166$ \\
\hline & $\mathrm{DE}$ & $1900.8 \pm 0.7848$ & $2586.6 \pm 0.7555$ & $993.90 \pm 0.5915$ & $5481.3 \pm 1.356$ & $2.5302 \pm 0.0263$ \\
\hline & PSO & $1898.7 \pm 11.56$ & $2587.3 \pm 0.3159$ & $989.85 \pm 9.183$ & $5475.9 \pm 16.11$ & $2.5318 \pm 0.0498$ \\
\hline & GSO & $1896.6 \pm 14.39$ & $2585.6 \pm 6.747$ & $979.21 \pm 15.64$ & $5461.4 \pm 20.90$ & $2.6557 \pm 0.0245$ \\
\hline & APSOW & $1901.7 \pm 0.2286$ & $2587.3 \pm 0.3411$ & $994.67 \pm 0.2273$ & $5483.7 \pm 0.5306$ & $2.6516 \pm 0.0352$ \\
\hline \multirow{5}{*}{ Gellybeans 1} & GA & $1071.5 \pm 8.708$ & $1957.0 \pm 11.28$ & $1694.2 \pm 10.43$ & $4722.7 \pm 9.509$ & $0.9959 \pm 0.0539$ \\
\hline & $\mathrm{DE}$ & $1089.4 \pm 0.6389$ & $1974.4 \pm 0.8169$ & $1718.0 \pm 1.982$ & $4781.9 \pm 3.067$ & $0.9589 \pm 0.0347$ \\
\hline & PSO & $1088.0 \pm 4.833$ & $1974.0 \pm 4.541$ & $1707.7 \pm 17.27$ & $4769.7 \pm 16.29$ & $0.9662 \pm 0.0422$ \\
\hline & GSO & $1082.9 \pm 8.614$ & $1971.0 \pm 7.501$ & $1707.7 \pm 15.16$ & $4761.7 \pm 22.17$ & $1.1298 \pm 0.0329$ \\
\hline & APSOW & $1089.8 \pm 0.1630$ & $1975.1 \pm 0.1775$ & $1718.8 \pm 0.1565$ & $4783.7 \pm 0.3207$ & $0.9839 \pm 0.0086$ \\
\hline \multirow{5}{*}{ Gellybeans2 } & GA & $1425.7 \pm 12.99$ & $2478.8 \pm 13.96$ & $2017.5 \pm 13.70$ & $5921.9 \pm 16.49$ & $0.9839 \pm 0.0266$ \\
\hline & $\mathrm{DE}$ & $1448.4 \pm 0.6094$ & $2496.3 \pm 0.4967$ & $2045.5 \pm 0.4752$ & $5990.2 \pm 0.9319$ & $0.9656 \pm 0.0305$ \\
\hline & PSO & $1446.2 \pm 6.556$ & $2494.7 \pm 7.948$ & $2034.1 \pm 14.91$ & $5974.9 \pm 20.87$ & $0.9563 \pm 0.0252$ \\
\hline & GSO & $1442.7 \pm 7.625$ & $2492.8 \pm 9.460$ & $2030.5 \pm 18.59$ & $5966.0 \pm 23.23$ & $1.1261 \pm 0.0355$ \\
\hline & APSOW & $1448.8 \pm 0.8718$ & $2496.6 \pm 0.3538$ & $2045.9 \pm 0.4971$ & $5991.3 \pm 1.382$ & $0.9876 \pm 0.0104$ \\
\hline \multirow{5}{*}{ House1 } & GA & $2386.6 \pm 28.13$ & $2197.9 \pm 25.78$ & $3593.3 \pm 22.10$ & $8177.8 \pm 34.24$ & $2.5531 \pm 0.0527$ \\
\hline & $\mathrm{DE}$ & $2437.1 \pm 0.9650$ & $2239.4 \pm 1.455$ & $3631.9 \pm 2.163$ & $8308.5 \pm 2.497$ & $2.5204 \pm 0.0190$ \\
\hline & PSO & $2436.2 \pm 12.56$ & $2241.0 \pm 0.3367$ & $3629.4 \pm 14.69$ & $8306.5 \pm 18.62$ & $2.5094 \pm 0.0113$ \\
\hline & GSO & $2438.2 \pm 0.8670$ & $2239.9 \pm 2.625$ & $3630.3 \pm 10.66$ & $8308.5 \pm 10.95$ & $2.6678 \pm 0.0338$ \\
\hline & APSOW & $2438.3 \pm 0.4987$ & $2240.8 \pm 0.4893$ & $3633.8 \pm 1.548$ & $8312.9 \pm 1.634$ & $2.6416 \pm 0.0209$ \\
\hline \multirow{5}{*}{ House 2} & GA & $919.25 \pm 13.07$ & $3027.5 \pm 12.75$ & $3855.7 \pm 12.03$ & $7802.5 \pm 14.86$ & $0.9793 \pm 0.0181$ \\
\hline & $\mathrm{DE}$ & $944.90 \pm 2.030$ & $3050.7 \pm 2.705$ & $3878.1 \pm 1.691$ & $7873.8 \pm 3.790$ & $0.9677 \pm 0.0328$ \\
\hline & PSO & $943.23 \pm 7.124$ & $3050.7 \pm 5.679$ & $3875.8 \pm 9.497$ & $7869.7 \pm 11.53$ & $0.9604 \pm 0.0201$ \\
\hline & GSO & $9421.6 \pm 6.878$ & $3050.0 \pm 6.484$ & $3871.1 \pm 21.74$ & $7863.3 \pm 23.27$ & $1.1296 \pm 0.0379$ \\
\hline & APSOW & $946.24 \pm 1.802$ & $3052.7 \pm 2.275$ & $3880.1 \pm 0.8102$ & $7879.0 \pm 3.184$ & $0.9875 \pm 0.0096$ \\
\hline \multirow{5}{*}{ Lake } & GA & $1703.5 \pm 20.82$ & $5823.2 \pm 20.14$ & $5748.2 \pm 23.15$ & $13275.0 \pm 21.76$ & $2.5479 \pm 0.0191$ \\
\hline & $\mathrm{DE}$ & $1741.1 \pm 1.420$ & $5866.6 \pm 1.050$ & $5795.3 \pm 1.454$ & $13403.0 \pm 2.507$ & $2.5370 \pm 0.0325$ \\
\hline & PSO & $1739.1 \pm 12.26$ & $5867.9 \pm 0.2356$ & $5794.6 \pm 10.27$ & $13401.6 \pm 15.57$ & $2.5094 \pm 0.0089$ \\
\hline & GSO & $1735.9 \pm 12.56$ & $5866.4 \pm 42.06$ & $5789.8 \pm 17.31$ & $13392.1 \pm 19.36$ & $2.6734 \pm 0.0488$ \\
\hline & APSOW & $1742.2 \pm 0.3352$ & $5867.7 \pm 0.3839$ & $5796.3 \pm 0.5681$ & $13406.1 \pm 0.9190$ & $2.6428 \pm 0.0258$ \\
\hline \multirow{5}{*}{ Lena } & GA & $2276.4 \pm 21.49$ & $2619.5 \pm 23.90$ & $1053.1 \pm 26.14$ & $5948.9 \pm 32.29$ & $2.5500 \pm 0.0282$ \\
\hline & $\mathrm{DE}$ & $2321.0 \pm 1.181$ & $2673.8 \pm 2.382$ & $1095.4 \pm 1.223$ & $6090.1 \pm 3.691$ & $2.5308 \pm 0.0693$ \\
\hline & PSO & $2312.1 \pm 20.23$ & $2665.1 \pm 26.81$ & $1090.0 \pm 18.49$ & $6067.1 \pm 38.18$ & $2.5115 \pm 0.0226$ \\
\hline & GSO & $2307.5 \pm 20.77$ & $2673.5 \pm 3.752$ & $1081.3 \pm 24.15$ & $6062.3 \pm 27.25$ & $2.6735 \pm 0.0534$ \\
\hline & APSOW & $2321.9 \pm 0.5466$ & $2675.2 \pm 0.4719$ & $1096.3 \pm 0.8450$ & $6093.4 \pm 1.555$ & $2.6395 \pm 0.0259$ \\
\hline \multirow{5}{*}{ Mandril } & GA & $2863.8 \pm 36.65$ & $2122.2 \pm 27.08$ & $3587.6 \pm 34.85$ & $8573.6 \pm 35.04$ & $2.5719 \pm 0.0257$ \\
\hline & $\mathrm{DE}$ & $2922.8 \pm 1.159$ & $2167.8 \pm 1.267$ & $3644.7 \pm 0.9718$ & $8735.4 \pm 1.853$ & $2.5407 \pm 0.0261$ \\
\hline & PSO & $2924.0 \pm 0.2345$ & $2160.0 \pm 21.13$ & $3639.4 \pm 22.74$ & $8723.5 \pm 28.99$ & $2.5406 \pm 0.0270$ \\
\hline & GSO & $2923.7 \pm 0.6716$ & $2165.6 \pm 10.78$ & $3645.2 \pm 0.8095$ & $8734.5 \pm 10.88$ & $2.7010 \pm 0.0826$ \\
\hline & APSOW & $2923.4 \pm 0.9006$ & $2169.4 \pm 0.3442$ & $3645.4 \pm 0.2333$ & $8738.2 \pm 1.080$ & $2.6593 \pm 0.0248$ \\
\hline \multirow{5}{*}{ Peppers } & GA & $1889.4 \pm 28.18$ & $5427.5 \pm 2.997$ & $1791.5 \pm 31.11$ & $9108.4 \pm 32.10$ & $2.5495 \pm 0.0193$ \\
\hline & $\mathrm{DE}$ & $1935.0 \pm 1033.7$ & $5478.2 \pm 1.240$ & $1847.1 \pm 1.705$ & $9260.2 \pm 2.699$ & $2.5646 \pm 0.0867$ \\
\hline & PSO & $1934.5 \pm 9.283$ & $5479.6 \pm 0.1274$ & $1836.0 \pm 33.09$ & $9250.2 \pm 33.72$ & $2.5260 \pm 0.0283$ \\
\hline & GSO & $1933.2 \pm 4.137$ & $5478.9 \pm 1.162$ & $1836.6 \pm 24.33$ & $9248.7 \pm 24.56$ & $2.6594 \pm 0.0255$ \\
\hline & APSOW & $1935.9 \pm 0.5011$ & $5479.2 \pm 0.4551$ & $1848.6 \pm 0.2473$ & $9263.7 \pm 0.8627$ & $2.7281 \pm 0.1791$ \\
\hline \multirow{5}{*}{ Splash } & GA & $3452.3 \pm 29.73$ & $3480.6 \pm 22.92$ & $2074.7 \pm 19.29$ & $9007.5 \pm 26.43$ & $2.5672 \pm 0.0623$ \\
\hline & $\mathrm{DE}$ & $3487.1 \pm 0.8918$ & $3520.3 \pm 6.159$ & $2116.7 \pm 1.767$ & $9124.1 \pm 6.798$ & $2.5370 \pm 0.0385$ \\
\hline & PSO & $3487.9 \pm 0.5831$ & $3514.5 \pm 19.68$ & $2117.0 \pm 4.905$ & $9119.4 \pm 20.86$ & $2.5255 \pm 0.0724$ \\
\hline & GSO & $3488.0 \pm 0.2391$ & $3503.2 \pm 19.60$ & $2112.6 \pm 10.46$ & $9103.8 \pm 23.65$ & $2.6818 \pm 0.0755$ \\
\hline & APSOW & $3487.9 \pm 0.1822$ & $3522.2 \pm 0.2994$ & $2117.8 \pm 0.1569$ & $9128.0 \pm 0.4447$ & $2.6510 \pm 0.0710$ \\
\hline \multirow{5}{*}{ Tiffany } & GA & $907.09 \pm 7.918$ & $1014.3 \pm 9.706$ & $525.00 \pm 10.83$ & $2446.4 \pm 10.72$ & $2.5480 \pm 0.0508$ \\
\hline & $\mathrm{DE}$ & $922.62 \pm 0.5295$ & $1029.3 \pm 0.4187$ & $543.85 \pm 2.059$ & $2495.8 \pm 1.923$ & $2.5224 \pm 0.0320$ \\
\hline & PSO & $921.16 \pm 9.121$ & $1029.2 \pm 0.7900$ & $539.79 \pm 10.34$ & $2490.1 \pm 12.99$ & $2.5026 \pm 0.0118$ \\
\hline & GSO & $909.96 \pm 20.28$ & $1028.8 \pm 1.717$ & $534.06 \pm 14.81$ & $2472.8 \pm 24.36$ & $2.6600 \pm 0.0374$ \\
\hline & APSOW & $922.83 \pm 0.1525$ & $1029.4 \pm 0.4738$ & $542.93 \pm 2.532$ & $2495.1 \pm 2.383$ & $2.6386 \pm 0.0430$ \\
\hline \multirow{5}{*}{ Tree } & GA & $3126.3 \pm 18.29$ & $5694.7 \pm 16.02$ & $3872.6 \pm 18.67$ & $12693.7 \pm 22.95$ & $0.9927 \pm 0.0220$ \\
\hline & $\mathrm{DE}$ & $3157.7 \pm 2.174$ & $5722.8 \pm 2.157$ & $3908.9 \pm 2.325$ & $12789.4 \pm 3.839$ & $0.9641 \pm 0.0211$ \\
\hline & PSO & $3153.8 \pm 10.42$ & $5724.8 \pm 1.073$ & $3905.5 \pm 9.540$ & $12784.0 \pm 14.83$ & $0.9490 \pm 0.0099$ \\
\hline & GSO & $3148.3 \pm 11.92$ & $5724.0 \pm 1.450$ & $3902.0 \pm 8.431$ & $12774.4 \pm 13.21$ & $1.1156 \pm 0.0179$ \\
\hline & APSOW & $3158.9 \pm 4.339$ & $5724.7 \pm 0.7861$ & $3909.8 \pm 3.073$ & $12793.4 \pm 5.359$ & $0.9969 \pm 0.0126$ \\
\hline
\end{tabular}


Tabela 5. Experimental results for 5-D: Mean \pm standard deviation.

\begin{tabular}{|c|c|c|c|c|c|c|}
\hline Image & Algorithm & $f_{I_{R}}$ & $f_{I_{G}}$ & $f_{I_{B}}$ & $f_{I}$ & Time $(\mathrm{s})$ \\
\hline \multirow{5}{*}{ Airplane } & GA & $1898.4 \pm 14.29$ & $2586.2 \pm 13.95$ & $977.68 \pm 14.14$ & $5462.4 \pm 15.64$ & $2.6348 \pm 0.0336$ \\
\hline & DE & $1921.3 \pm 1.349$ & $2616.3 \pm 2.035$ & $1001.7 \pm 3.091$ & $5539.4 \pm 4.710$ & $2.6083 \pm 0.0238$ \\
\hline & PSO & $1920.6 \pm 6.426$ & $2614.5 \pm 10.24$ & $1000.4 \pm 7.258$ & $5535.6 \pm 13.42$ & $2.6068 \pm 0.0184$ \\
\hline & GSO & $1919.2 \pm 8.976$ & $2611.8 \pm 11.01$ & $994.30 \pm 11.65$ & $5525.4 \pm 16.82$ & $2.7636 \pm 0.0195$ \\
\hline & APSOW & $1922.5 \pm 0.7313$ & $2618.1 \pm 0.7350$ & $1003.9 \pm 3.481$ & $5544.5 \pm 3.675$ & $2.7536 \pm 0.0518$ \\
\hline \multirow{5}{*}{ Gellybeans1 } & GA & $1082.9 \pm 7.351$ & $1971.7 \pm 10.17$ & $1711.0 \pm 10.54$ & $4765.7 \pm 10.69$ & $1.0515 \pm 0.0250$ \\
\hline & $\mathrm{DE}$ & $1094.8 \pm 1.765$ & $1986.9 \pm 0.5476$ & $1729.6 \pm 0.7525$ & $4811.4 \pm 1.890$ & $1.0349 \pm 0.0267$ \\
\hline & PSO & $1092.0 \pm 5.317$ & $1985.7 \pm 4.230$ & $1721.7 \pm 11.02$ & $4799.5 \pm 13.23$ & $1.0261 \pm 0.0236$ \\
\hline & GSO & $1089.6 \pm 5.827$ & $1983.0 \pm 6.324$ & $1716.6 \pm 15.12$ & $4789.3 \pm 15.69$ & $1.2010 \pm 0.0273$ \\
\hline & APSOW & $1095.4 \pm 0.5348$ & $1986.7 \pm 2.284$ & $1727.5 \pm 4.987$ & $4809.8 \pm 5.048$ & $1.0506 \pm 0.0079$ \\
\hline \multirow{5}{*}{ Gellybeans2 } & GA & $1441.4 \pm 8.232$ & $2495.2 \pm 11.62$ & $2038.8 \pm 11.94$ & $5975.6 \pm 16.28$ & $1.0489 \pm 0.0195$ \\
\hline & $\mathrm{DE}$ & $1456.8 \pm 2.182$ & $2514.8 \pm 1.106$ & $2061.5 \pm 4.977$ & $6033.2 \pm 6.744$ & $1.0287 \pm 0.0221$ \\
\hline & PSO & $1455.4 \pm 4.696$ & $2512.7 \pm 7.402$ & $2054.2 \pm 8.927$ & $6022.4 \pm 12.91$ & $1.0224 \pm 0.0200$ \\
\hline & GSO & $1451.3 \pm 7.218$ & $2511.9 \pm 7.239$ & $2051.4 \pm 12.47$ & $6014.6 \pm 13.20$ & $1.1969 \pm 0.0285$ \\
\hline & APSOW & $1458.4 \pm 0.7110$ & $2515.4 \pm 3.563$ & $2059.7 \pm 7.227$ & $6033.6 \pm 9.179$ & $1.0625 \pm 0.0352$ \\
\hline \multirow{5}{*}{ House1 } & GA & $2427.7 \pm 21.99$ & $2235.3 \pm 21.87$ & $3634.4 \pm 20.64$ & $8297.5 \pm 25.37$ & $2.6224 \pm 0.0247$ \\
\hline & DE & $2467.7 \pm 1.733$ & $2285.6 \pm 1.876$ & $3676.0 \pm 2.019$ & $8429.4 \pm 3.274$ & $2.6073 \pm 0.0219$ \\
\hline & PSO & $2465.3 \pm 10.79$ & $2281.4 \pm 14.91$ & $3676.8 \pm 7.688$ & $8423.6 \pm 17.55$ & $2.616 \pm 0.0649$ \\
\hline & GSO & $2463.9 \pm 9.188$ & $2280.4 \pm 12.62$ & $3668.4 \pm 17.11$ & $8412.9 \pm 21.46$ & $2.7776 \pm 0.0494$ \\
\hline & APSOW & $2469.4 \pm 0.6580$ & $2287.0 \pm 0.6757$ & $3677.8 \pm 0.8035$ & $8434.2 \pm 1.449$ & $2.7333 \pm 0.0191$ \\
\hline \multirow{5}{*}{ House2 } & GA & $938.02 \pm 11.45$ & $3050.5 \pm 14.99$ & $3872.1 \pm 13.17$ & $7860.7 \pm 13.53$ & $1.0615 \pm 0.0331$ \\
\hline & DE & $966.72 \pm 3.789$ & $3076.9 \pm 3.162$ & $3893.5 \pm 1.717$ & $7937.2 \pm 6.481$ & $1.0781 \pm 0.0811$ \\
\hline & PSO & $960.59 \pm 12.64$ & $3078.2 \pm 21.12$ & $3890.6 \pm 8.959$ & $7929.4 \pm 15.41$ & $1.0651 \pm 0.0726$ \\
\hline & GSO & $957.45 \pm 11.99$ & $3077.6 \pm 2.164$ & $3885.7 \pm 10.69$ & $7920.8 \pm 16.86$ & $1.1974 \pm 0.0467$ \\
\hline & APSOW & $968.41 \pm 0.8029$ & $3078.9 \pm 1.505$ & $3894.6 \pm 2.723$ & $7941.9 \pm 3.029$ & $1.0542 \pm 0.0091$ \\
\hline \multirow{5}{*}{ Lake } & GA & $1724.9 \pm 23.78$ & $5880.0 \pm 20.81$ & $5787.7 \pm 19.18$ & $13392.7 \pm 20.43$ & $2.6240 \pm 0.0115$ \\
\hline & $\mathrm{DE}$ & $1768.5 \pm 1.605$ & $5917.1 \pm 1.429$ & $5828.0 \pm 1.693$ & $13513.7 \pm 2.730$ & $2.8870 \pm 0.1690$ \\
\hline & PSO & $1766.8 \pm 8.546$ & $5913.4 \pm 15.58$ & $5823.5 \pm 1.370$ & $13503.8 \pm 21.58$ & $2.8792 \pm 0.1447$ \\
\hline & GSO & $1760.6 \pm 12.77$ & $5916.3 \pm 4.290$ & $5822.4 \pm 11.46$ & $13499.4 \pm 17.14$ & $2.7734 \pm 0.0556$ \\
\hline & APSOW & $1769.8 \pm 0.8341$ & $5918.4 \pm 0.6596$ & $5830.0 \pm 0.8718$ & $13518.3 \pm 1.588$ & $2.7521 \pm 0.0933$ \\
\hline \multirow{5}{*}{ Lena } & GA & $2308.7 \pm 22.49$ & $2672.6 \pm 20.11$ & $1076.8 \pm 18.21$ & $6058.3 \pm 19.53$ & $2.6308 \pm 0.0244$ \\
\hline & $\mathrm{DE}$ & $2336.0 \pm 6.397$ & $2707.1 \pm 1.958$ & $1113.9 \pm 2.264$ & $6157.1 \pm 6.803$ & $2.7755 \pm 0.0869$ \\
\hline & PSO & $2333.5 \pm 9.541$ & $2703.4 \pm 12.83$ & $1107.8 \pm 9.628$ & $6144.8 \pm 18.53$ & $2.7531 \pm 0.0880$ \\
\hline & GSO & $2320.4 \pm 20.14$ & $2704.7 \pm 9.857$ & $1100.1 \pm 14.24$ & $6125.3 \pm 26.45$ & $2.7776 \pm 0.0419$ \\
\hline & APSOW & $2340.1 \pm 3.977$ & $2708.5 \pm 0.6935$ & $1114.2 \pm 4.086$ & $6162.9 \pm 6.271$ & $2.7322 \pm 0.0608$ \\
\hline \multirow{5}{*}{ Mandril } & GA & $2920.4 \pm 31.67$ & $2156.4 \pm 19.95$ & $3640.0 \pm 22.86$ & $8716.9 \pm 29.53$ & $2.6557 \pm 0.0388$ \\
\hline & $\mathrm{DE}$ & $2967.6 \pm 2.762$ & $2199.8 \pm 1.980$ & $3690.7 \pm 1.822$ & $8858.2 \pm 3.931$ & $2.7917 \pm 0.0673$ \\
\hline & PSO & $2970.7 \pm 0.8978$ & $2196.8 \pm 11.38$ & $3685.1 \pm 18.08$ & $8852.7 \pm 19.48$ & $2.7942 \pm 0.0348$ \\
\hline & GSO & $2969.6 \pm 2.888$ & $2195.4 \pm 12.39$ & $3692.5 \pm 1.869$ & $8857.7 \pm 13.50$ & $2.8016 \pm 0.0684$ \\
\hline & APSOW & $2970.5 \pm 1.146$ & $2201.7 \pm 0.9516$ & $3692.6 \pm 0.9582$ & $8865.0 \pm 1.946$ & $2.7625 \pm 0.0465$ \\
\hline \multirow{5}{*}{ Peppers } & GA & $1924.5 \pm 20.50$ & $5485.3 \pm 18.68$ & $1834.9 \pm 18.03$ & $9244.7 \pm 23.55$ & $2.6458 \pm 0.0497$ \\
\hline & $\mathrm{DE}$ & $1963.9 \pm 1.940$ & $5523.2 \pm 1.897$ & $1877.5 \pm 2.268$ & $9364.7 \pm 4.274$ & $2.6062 \pm 0.0112$ \\
\hline & PSO & $1961.9 \pm 10.25$ & $5518.9 \pm 14.74$ & $1869.8 \pm 14.76$ & $9350.8 \pm 24.21$ & $2.6328 \pm 0.1370$ \\
\hline & GSO & $1957.9 \pm 15.28$ & $5523.8 \pm 2.094$ & $1872.6 \pm 10.93$ & $9354.4 \pm 19.77$ & $2.7906 \pm 0.0711$ \\
\hline & APSOW & $1965.8 \pm 0.6985$ & $5524.0 \pm 1.121$ & $1880.3 \pm 0.6652$ & $9370.2 \pm 1.775$ & $2.7307 \pm 0.0199$ \\
\hline \multirow{5}{*}{ Splash } & GA & $3480.3 \pm 15.39$ & $3523.7 \pm 14.23$ & $2100.6 \pm 17.24$ & $9104.6 \pm 19.32$ & $2.6740 \pm 0.1000$ \\
\hline & $\mathrm{DE}$ & $3507.3 \pm 2.704$ & $3547.7 \pm 2.135$ & $2129.5 \pm 2.568$ & $9184.6 \pm 3.391$ & $2.6146 \pm 0.0531$ \\
\hline & PSO & $3507.9 \pm 5.747$ & $3548.8 \pm 5.480$ & $2129.0 \pm 6.296$ & $9185.8 \pm 10.00$ & $2.6031 \pm 0.0709$ \\
\hline & GSO & $3504.8 \pm 7.828$ & $3545.9 \pm 8.745$ & $2129.7 \pm 2.605$ & $9180.6 \pm 12.88$ & $2.7635 \pm 0.0306$ \\
\hline & APSOW & $3510.3 \pm 0.9714$ & $3550.6 \pm 2.150$ & $2132.0 \pm 3.272$ & $9193.0 \pm 5.064$ & $2.7157 \pm 0.0087$ \\
\hline \multirow{5}{*}{ Tiffany } & GA & $919.23 \pm 9.068$ & $1047.6 \pm 8.907$ & $540.53 \pm 11.27$ & $2507.3 \pm 9.670$ & $2.6302 \pm 0.0230$ \\
\hline & $\mathrm{DE}$ & $933.71 \pm 3.807$ & $1063.9 \pm 2.278$ & $563.06 \pm 0.7680$ & $2560.7 \pm 4.205$ & $2.6047 \pm 0.0137$ \\
\hline & PSO & $930.59 \pm 8.803$ & $1061.7 \pm 1.089$ & $562.80 \pm 4.246$ & $2555.1 \pm 17.41$ & $2.5895 \pm 0.0123$ \\
\hline & GSO & $923.90 \pm 13.02$ & $1056.2 \pm 13.09$ & $553.29 \pm 9.277$ & $2533.4 \pm 18.52$ & $2.7682 \pm 0.0477$ \\
\hline & APSOW & $934.70 \pm 4.336$ & $1065.2 \pm 0.2536$ & $563.50 \pm 0.4289$ & $2563.4 \pm 4.467$ & $2.718 \pm 0.0081$ \\
\hline \multirow{5}{*}{ Tree } & GA & $3160.0 \pm 12.45$ & $5727.5 \pm 16.93$ & $3899.5 \pm 17.78$ & $12787.0 \pm 16.92$ & $1.0849 \pm 0.0298$ \\
\hline & $\mathrm{DE}$ & $3181.7 \pm 1.335$ & $5765.0 \pm 1.834$ & $3929.4 \pm 1.728$ & $12876.2 \pm 2.722$ & $1.0360 \pm 0.0357$ \\
\hline & PSO & $3181.2 \pm 5.859$ & $5764.0 \pm 10.59$ & $3926.3 \pm 9.951$ & $12871.5 \pm 16.07$ & $1.0292 \pm 0.0240$ \\
\hline & GSO & $3182.9 \pm 1.448$ & $5761.0 \pm 12.54$ & $3930.0 \pm 4.199$ & $12874.1 \pm 12.84$ & $1.2016 \pm 0.0179$ \\
\hline & APSOW & $3181.9 \pm 4.258$ & $5766.5 \pm 0.6905$ & $3931.7 \pm 1.166$ & $12880.3 \pm 4.494$ & $1.0599 \pm 0.0083$ \\
\hline
\end{tabular}


Tabela 6. Overall Evaluation: Average Ranks for the Friedman Test, with a critical distance $C D=1.7608$.

\begin{tabular}{|r|c|c|c|c|}
\hline \multicolumn{5}{|c|}{$2-\mathrm{D}$} \\
\hline Algorithm & $f_{I_{R}}$ & $f_{I_{G}}$ & $f_{I_{B}}$ & $f_{I}$ \\
\hline GA & 18.4958 & 17.7833 & 17.0750 & 17.5083 \\
DE & 91.2903 & 88.7222 & 88.3625 & $\mathbf{9 5 . 2 4 0 3}$ \\
PSO & 88.1458 & 88.9806 & $\mathbf{9 1 . 3 9 7 2}$ & 86.1708 \\
GSO & 84.8153 & 86.0931 & 89.3347 & 82.5542 \\
APSOW & $\mathbf{9 4 . 7 5 2 8}$ & $\mathbf{9 5 . 9 2 0 8}$ & $\mathbf{9 1 . 3 3 0 6}$ & $\mathbf{9 6 . 0 2 6 4}$ \\
\hline \multicolumn{5}{|c|}{$3-\mathrm{D}$} \\
\hline Algorithm & $f_{I_{R}}$ & $f_{I_{G}}$ & $f_{I_{B}}$ & $f_{I}$ \\
\hline GA & 19.5750 & 17.3472 & 20.5778 & 17.2694 \\
DE & 72.4042 & 67.6625 & 74.1125 & 77.5958 \\
PSO & $\mathbf{1 0 0 . 4 9 8 6}$ & $\mathbf{1 0 7 . 9 7 3 6}$ & 97.9556 & 101.7583 \\
GSO & 84.4417 & 91.3319 & 83.6264 & 75.1444 \\
APSOW & $\mathbf{1 0 0 . 5 8 0 6}$ & 93.1847 & $\mathbf{1 0 1 . 2 2 7 8}$ & $\mathbf{1 0 5 . 7 3 1 9}$ \\
\hline \multicolumn{5}{|c|}{$4-\mathrm{D}$} \\
\hline Algorithm & $f_{I_{R}}$ & $f_{I_{G}}$ & $f_{I_{B}}$ & $f_{I}$ \\
\hline GA & 19.3917 & 18.2069 & 22.2167 & 16.6333 \\
DE & 71.1847 & 67.4778 & 76.7292 & 80.8486 \\
PSO & $\mathbf{1 0 5 . 4 9 8 6}$ & $\mathbf{1 0 6 . 5 0 1 4}$ & 97.0500 & 98.5611 \\
GSO & 81.6278 & 85.5736 & 78.7264 & 68.6472 \\
APSOW & 99.7972 & 99.7403 & $\mathbf{1 0 2 . 7 7 7 8}$ & $\mathbf{1 1 2 . 8 0 9 7}$ \\
\hline \multicolumn{5}{|c|}{$5-\mathrm{D}$} \\
\hline Algorithm & $f_{I_{R}}$ & $f_{I_{G}}$ & $f_{I_{B}}$ & $f_{I}$ \\
\hline GA & 21.5556 & 19.4778 & 21.8556 & 16.4778 \\
DE & 77.1069 & 74.1931 & 80.7833 & 87.3750 \\
PSO & 95.8583 & $\mathbf{1 0 0 . 4 6 2 5}$ & 93.1431 & 88.2361 \\
GSO & 76.1528 & 83.0236 & 75.9778 & 67.6722 \\
APSOW & $\mathbf{1 0 6 . 8 2 6 4}$ & $\mathbf{1 0 0 . 3 4 3 1}$ & $\mathbf{1 0 5 . 7 4 0 3}$ & $\mathbf{1 1 7 . 7 3 8 9}$ \\
\hline
\end{tabular}

As future works, we intend to adapt some PSO variants to the context of image segmentation by multi-level thresholding. We also intend to hybridize PSO with other EAs from the literature to perform image segmentation.

\section{Acknowledgment}

The authors would like to thank FACEPE, CNPq and CAPES (Brazilian Research Agencies) for their financial support.

\section{Referências}

Demšar, J. (2006). Statistical comparisons of classifiers over multiple data sets. The Journal of Machine Learning Research, 7:1-30.

Elaziz, M. A., Bhattacharyya, S., and Lu, S. (2019). Swarm selection method for multilevel thresholding image segmentation. Expert Systems with Applications, 138:112818.

Friedman, M. (1937). The use of ranks to avoid the assumption of normality implicit in the analysis of variance. Journal of the american statistical association, 32(200):675701.

He, S., Wu, Q. H., and Saunders, J. (2009). Group search optimizer: an optimization algorithm inspired by animal searching behavior. Evolutionary Computation, IEEE Transactions on, 13(5):973-990.

Holland, J. H. (1992). Genetic algorithms. Scientific american, 267(1):66-72. 
Kennedy, J. and Eberhart, R. (1995). Particle swarm optimization. In Neural Networks, 1995. Proceedings., IEEE International Conference on, volume 4, pages 1942-1948. IEEE.

Kennedy, J., Eberhart, R. C., and Shi, Y. (2001). Swarm intelligence. 2001. Kaufmann, San Francisco.

Kumar, S., Pant, M., and Ray, A. (2011). Differential evolution embedded otsu's method for optimized image thresholding. In Information and Communication Technologies (WICT), 2011 World Congress on, pages 325-329. IEEE.

Liu, D. and Yu, J. (2009). Otsu method and k-means. In Hybrid Intelligent Systems, 2009. HIS'09. Ninth International Conference on, volume 1, pages 344-349. IEEE.

Otsu, N. (1979). A threshold selection method from gray-level histograms. IEEE transactions on systems, man, and cybernetics, 9(1):62-66.

Pacifico, L. D. S., Ludermir, T. B., and Britto, L. F. S. (2018). A hybrid improved group search optimization and otsu method for color image segmentation. In 2018 7th Brazilian Conference on Intelligent Systems (BRACIS), pages 296-301. IEEE.

Parthasarathy, G. and Chitra, D. (2015). Thresholding technique for color image segmentation. International Journal for Research in Applied Science \& Engineering Technology, 3(6):437-445.

Rodríguez-Esparza, E., Zanella-Calzada, L. A., Oliva, D., Heidari, A. A., Zaldivar, D., Pérez-Cisneros, M., and Foong, L. K. (2020). An efficient harris hawks-inspired image segmentation method. Expert Systems with Applications, page 113428.

Storn, R. and Price, K. (1997). Differential evolution-a simple , efficient heuristic for global optimization over continuous spaces. Journal of global optimization, 11(4):341359.

Suresh, S. and Lal, S. (2017). Multilevel thresholding based on chaotic darwinian particle swarm optimization for segmentation of satellite images. Applied Soft Computing, 55:503-522.

Wang, Y. and Tan, Z. (2019). Multilevel image thresholding based on adaptive particle swarm optimization. In 2019 International Conference on Intelligent Computing, Automation and Systems (ICICAS), pages 634-637. IEEE.

Ye, Z., Ma, L., Zhao, W., Liu, W., and Chen, H. (2015). A multi-level thresholding approach based on group search optimization algorithm and otsu. In Computational Intelligence and Design, 2006. CEC 2006. IEEE International Symposium on, pages 275-278. IEEE.

Zhang, Z. and Zhou, N. (2012). A novel image segmentation method combined otsu and improved pso. In Advanced Computational Intelligence (ICACI), 2012 IEEE Fifth International Conference on, pages 583-586. IEEE.

Zhou, S. and Yang, P. (2011). Infrared image segmentation based on otsu and genetic algorithm. In Multimedia Technology (ICMT), 2011 International Conference on, pages 5421-5424. IEEE. 